\title{
Basic Study of Smart Robotic Construction Lift For Increasing Resource Lifting Efficiency in High-Rise Building Construction
}

\author{
Chang-Yeon, Cho ${ }^{1}$; Soon-Wook, Kwon ${ }^{2}$; Junbok Lee ${ }^{3}$, Suk-Jun,You ${ }^{4}$ Sang-Yoon-Chin ${ }^{5}$ and Yea-Sang, Kim ${ }^{6}$ \\ ${ }^{1}$ Dept. of Architecture \& Civil Engineering, Sungkyunkwan University,Suwon-si, Korea, P.O. Box 440-746, \\ PH(82)31-299-4772 ; FAX(82)31-290-7570 ; e-mail :yms09d@hanmail.com \\ 2 Dept. of Architecture \& Civil Engineering, Sungkyunkwan University,Suwon-si, Korea, P.O. Box 440-746, \\ PH(82)31-299-4765 ; FAX(82)31-290-7570 ; e-mail :swkwon@skku.edu \\ ${ }^{3}$ College of Architecture \& Civil Engineering, Kyung Hee University, Yongin-si, Korea, P.O. Box 449-701, \\ PH(82)31-201-2541 ; FAX(82)31-204-6780 ; e-mail : leejb@khu.ac.kr \\ ${ }^{4}$ Dept. of Architecture \& Civil Engineering, Sungkyunkwan University,Suwon-si, Korea, P.O. Box 440-746, \\ PH(82)31-299-4772 ; FAX(82)31-290-7570 ; e-mail : seokjoon.you@gmail.com \\ 5 Dept. of Architecture \& Civil Engineering, Sungkyunkwan University,Suwon-si, Korea, P.O. Box 440-746, \\ PH(82)31-290-7568 ; FAX(82)31-290-7570 ; e-mail : schin@skku.edu \\ ${ }^{6}$ Dept. of Architecture \& Civil Engineering, Sungkyunkwan University,Suwon-si, Korea, P.O. Box 440-746, \\ PH(82)31-290-7561 ; FAX(82)31-290-7570 ; e-mail :yeakim@skku.edu
}

\begin{abstract}
Most high-rise buildings are erected in downtown areas, where on-site storage space for construction materials is typically insufficient due to limited spaces. Just-In-Time (or JIT) concept has been adopted so that necessary stock materials and storage spaces can be reduced; for high-rise buildings, however, transporting materials vertically using lifts still poses significant efficiency because its efficiency drops exponentially as the building height grows.

Most of current efforts to counter this problem mainly focus on developing smart tower cranes, whereas lift has gathered less attention in this respect.

This research aims to develop a robotic lift capable of autonomous operations at night time, based on the intelligent lift development toolkit which was previously developed by the authors. It is now in a preliminary study phase.

This study proposes a concept model of the robotic lift system and its operation plans, utilizing several technologies such as an optimized material dispatch algorithm and ubiquitous sensor networks (USNs). The proposed system can be relayed to the horizontal transportation robots located in each floor to move the lifted materials to their destinations.

The outcome from this study will contribute to the improvement of the overall efficiency of the high-rise building construction logistics and space constraints of the construction site. It is also expected that the mechanical performance of the existing lift system can be benefited from this research.

When overall system development, including optimized operation planning model and monitoring subsystem, is finished, it will contribute to innovation of the construction technology.
\end{abstract}

Keywords. Smart Lift, Robotic Construction Lift, High-rise building construction, operation optimization, concept model

\section{Introduction and Motivation}

According to 'Skyscraper' webzine, around the globe there are so many ongoing highrise building projects, and competition among the new economic giants such as China and Dubai for the tallest building is apparent. The examples include Chicago Spiral building (150 floors, 610m), Ablaza Al Bite building (76 floors, 595m), Russia's Federation Tower (93 floors, 506m), World Finance Center in Shanghai (101 floors, 492m), etc.

According to an earlier research (Lee et al. 2008 ), height of a building in construction affects efficiency of lifting equipments installed on site, which also affects the overall schedule. Current generation of the lifts fall short of meeting the field needs, because they aren't fast enough (typically $100-150 \mathrm{~m} / \mathrm{min}$ ) to elevate 
materials to the desired floor on time if the floor is located above $300 \mathrm{~m}$; as a result, overall schedule is significantly affected from it.

To counter the efficiency issue, various techniques from working overtime to Six Sigma have been applied, only to reveal other issues such as workplace safety and optimization problems due to spatial and temporal constraints.

This research aims to develop fundamental concepts for next generation lift systems which allow higher efficiency and better management using intelligent control. To achieve this goal, the authors have analyzed existing lift systems with respect to their feasibility for high-rise application.

\section{Construction Lift (Literature Reviews)}

\subsection{Classification of the lift systems}

There are several different lift types commonly used in construction sites: first, construction hoists which move materials and men vertically; second, simpler ones which resemble passenger elevators and used for smaller loads; third, hydraulic ones. Construction lifts may be further sub-categorized into a low speed type, a medium speed type, a high speed type; a high capacity type, and special types. These types can be briefly described as follows:

a. Low-speed type: most commonly used in construction sites. Suitable for lower-rise buildings such as community housing and office buildings. Typical speed for this type is $38 \mathrm{~m} / \mathrm{min}$, and maximum lifting height is typically $150 \mathrm{~m}$; also, its nominal load is $1.0 \sim 1.2$ tons.

b. Medium-speed type: Suitable for medium-to-high rise projects. Hoists of this type generally have better loading capacity than the low-speed ones. Their maximum installable hight is $150 \mathrm{~m}$ to $300 \mathrm{~m}$, and their nominal load is 1.5 tons to 2.0 tons.

c. High capacity type: designed to increase lifting efficiency in mid-to-high rise projects. Its speed is virtually same as the low speed type, but can be installed to higher places (maximum installation height: $150 \mathrm{~m}$ to $200 \mathrm{~m}$ ), with increased nominal load (1.2 tons to 2.0 tons).

d. High speed type: designed specifically for high-rise projects. Its speed is $100 \mathrm{~m} / \mathrm{min}$, maximum installation height is at least 350 meters, and nominal load is 2.5 tons to 3.0 tons.

Table 1. Summarizes the performance of these lift types. Seen in the table, their mechanical performance differences come from the use of inverters, and location of the drive motors.

\begin{tabular}{|c|c|c|c|c|c|c|c|c|}
\hline types & $\begin{array}{c}\text { Nomina } \\
\text { loads } \\
\text { (ton) }\end{array}$ & $\begin{array}{c}\text { Lifting } \\
\text { speed } \\
(\mathrm{m} / \mathrm{min} \\
)\end{array}$ & $\begin{array}{c}\text { dimension } \\
\text { of the } \\
\text { loading } \\
\text { room } \\
(\mathrm{m})\end{array}$ & $\begin{array}{c}\text { Max. size } \\
\text { of the } \\
\text { door } \\
\text { opening(m } \\
)\end{array}$ & $\begin{array}{c}\text { Frequenc } \\
\text { y of } \\
\text { power } \\
\text { alternatio } \\
\mathrm{n} \\
(\mathrm{Hz})\end{array}$ & $\begin{array}{c}\text { Nominal } \\
\text { power } \\
\text { consumptio } \\
\mathrm{n} \\
(\mathrm{KVA})\end{array}$ & $\begin{array}{c}\text { Locatio } \\
\text { n of the } \\
\text { drive } \\
\text { motors }\end{array}$ & $\begin{array}{c}\text { Use of } \\
\text { inverter } \\
\mathrm{s}\end{array}$ \\
\hline $\begin{array}{c}\text { Low } \\
\text { speed }\end{array}$ & $1.0-1.2$ & 38 & $\begin{array}{c}1.27 \times 2.9 \times 2 . \\
5\end{array}$ & $1.27 \times 2.4$ & $50 / 60$ & $2 \times 27 \mathrm{KVA}$ & internal & no \\
\hline $\begin{array}{c}\text { Med } \\
\text { speed }\end{array}$ & $1.2-1.5$ & 70 & $\begin{array}{c}1.5 \times 4.0 \times 2.6 \\
5\end{array}$ & $1.5 \times 2.6$ & $50 / 60$ & $2 \times 47 \mathrm{KVA}$ & rooftop & yes \\
\hline $\begin{array}{c}\text { High } \\
\text { capacit } \\
\mathrm{y}\end{array}$ & $1.2-2.0$ & 38 & $\begin{array}{c}1.5 \times 4.0 \times 2.6 \\
5\end{array}$ & $1.5 \times 2.6$ & $50 / 60$ & $2 \times 75 \mathrm{KVA}$ & rooftop & yes \\
\hline $\begin{array}{c}\text { High } \\
\text { speed }\end{array}$ & $2.0-3.0$ & 100 & $\begin{array}{c}1.5 \times 4.5 \times 2.6 \\
5\end{array}$ & $1.5 \times 2.6$ & $50 / 60$ & $2 \times 64 \mathrm{KVA}$ & rooftop & yes \\
\hline
\end{tabular}

\subsection{Mechanical performance of construction hoists and survey of existing}

Figure 1 illustrates the nomenclature of a typical construction hoist. It is mainly composed of a loading cage (which actually move people and materials vertically), a mast which sustains the cage, a rack-and-pinion gear assembly which converts rotational force to vertical movement (for driving the cage), and a 
counterweight system. Details of the drive train are illustrated in Figure 2.
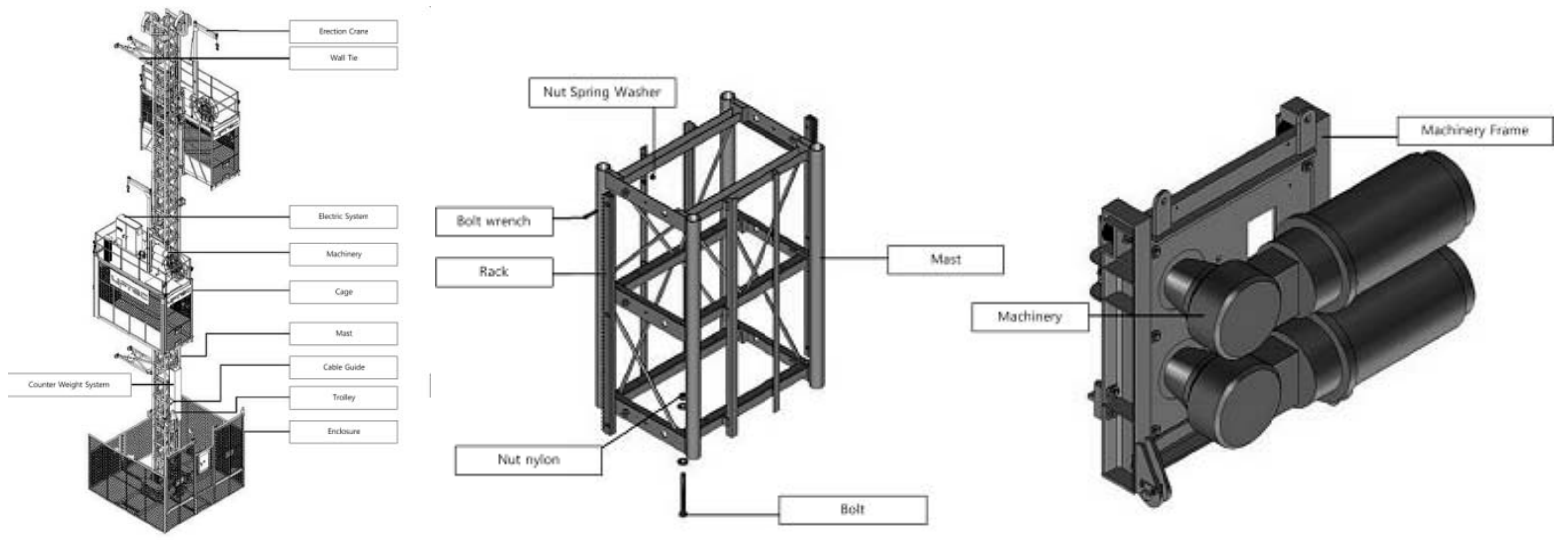

Figure 1. Typical Construction Lifts and Details of the Drive Train

A hoist can determine where it is located by counting teeth of the pinion gear while it is rotating, which can be adjusted with magnetic markers placed in each floor.

\subsection{Survey of precedent researches}

For the authors, it was unable to find any known researches in (at least in Journals of ASCE and Automation in Construction- the search was by no means exhaustive, as some undisclosed technical reports or regional research articles might have been omitted); on the other hand, most researches for automated material movement focused on tower cranes and construction robots. Majority of domestic researches in South Korea appeared to discuss management techniques for lifting efficiency rather than development of new equipments. They are summarized as follows:

(Lee JB and Han CH, 2008) evaluated economic feasibility of using medium speed hoists in buildings of 30-40 floors. (Chung et. al, 2004) suggested pre-fabrication and carefully planned load-balancing for lessening hoist workloads. In (Lee et. al, 2004), as a part of enterprise resource planning, improved hoist scheduling was proposed to implement just-in-time delivery. (Kim and Han, 2008) proposed a processof determining optimum number of hoists needed in systematic manner. (Park et. al, 2001) discussed optimization process of the hoist schedules for finish works.

\section{As-is Problem Statements}

\subsection{Analysis of of daily hoist travels with respect to lifting height}

In our previous research, efficiency of a hoist can be measured by the time needed to lift things to the designated height, whose approximate value can be calculated using the following expression:

\section{Time needed to lift $=\{$ average lifting height $(\mathrm{m}) /$ lifting speed $(\mathrm{m} / \mathrm{min}) \mathrm{x} 2\}+$ lead time $($ for loading and unloading)}

Table 2 shows the number of estimated single lift times and total number of daily travels of different hoist types if they were installed in Burj Al Arab Hotel project, which is 321 meters high. In this table, daiy work time is estimated to 8 hours, and Machines are operated in $70 \%$ to their full capability. 
Table 2. The number of estimated single lift times and total number of daily travels of different hoist types

\begin{tabular}{|c|c|c|c|c|c|c|c|}
\hline \multirow{2}{*}{$\begin{array}{c}\text { Lifting } \\
\text { height } \\
(\mathrm{m})\end{array}$} & $\begin{array}{c}\text { Lead } \\
\text { time } \\
(\mathrm{min})\end{array}$ & $\begin{array}{c}\text { Time of } \\
\text { single lift } \\
\text { (min) }\end{array}$ & $\begin{array}{c}\text { Number of } \\
\text { daily travels } \\
\text { (travels/day) }\end{array}$ & $\begin{array}{c}\text { Time of } \\
\text { single lift } \\
\text { (min) }\end{array}$ & $\begin{array}{c}\text { Number of } \\
\text { daily travels } \\
\text { (travels/day) }\end{array}$ & $\begin{array}{c}\text { Time of } \\
\text { single lift } \\
\text { (min) }\end{array}$ & $\begin{array}{c}\text { Number of } \\
\text { daily travels } \\
\text { (travels/day) }\end{array}$ \\
\hline 20 & 10 & 11.05 & 31 & 10.57 & 32 & 32.00 & 33 \\
\hline 40 & 10 & 12.11 & 28 & 11.14 & 31 & 31.00 & 32 \\
\hline 60 & 10 & 13.16 & 26 & 11.71 & 29 & 29.00 & 31 \\
\hline 80 & 10 & 14.21 & 24 & 12.29 & 28 & 28.00 & 30 \\
\hline 100 & 10 & 15.26 & 23 & 12.86 & 27 & 27.00 & 29 \\
\hline 120 & 10 & 16.32 & 21 & 13.43 & 26 & 26.00 & 28 \\
\hline 140 & 10 & 17.37 & 20 & 14.00 & 24 & 24.00 & 28 \\
\hline 160 & 10 & 18.42 & 19 & 14.57 & 24 & 24.00 & 27 \\
\hline 180 & 10 & 19.47 & 18 & 15.14 & 23 & 23.00 & 26 \\
\hline 200 & 10 & 20.53 & 17 & 15.71 & 22 & 22.00 & 26 \\
\hline 220 & 10 & 21.58 & 16 & 16.29 & 21 & 21.00 & 25 \\
\hline 240 & 10 & 22.63 & 15 & 16.86 & 20 & 20.00 & 24 \\
\hline 260 & 10 & 23.68 & 15 & 17.43 & 20 & 20.00 & 24 \\
\hline 280 & 10 & 24.74 & 14 & 18.00 & 19 & 19.00 & 23 \\
\hline 300 & 10 & 25.79 & 14 & 18.57 & 19 & 19.00 & 23 \\
\hline 320 & 10 & 26.84 & 13 & 19.14 & 18 & 18.00 & 22 \\
\hline & & & & & & & \\
\hline
\end{tabular}

\section{Time needed to lift with respect to lifting height}

....... low speed type Time needed to lift (min)

high speed type Time needed to lift

(min)
_ - medium speed type Time needed to lift (min)

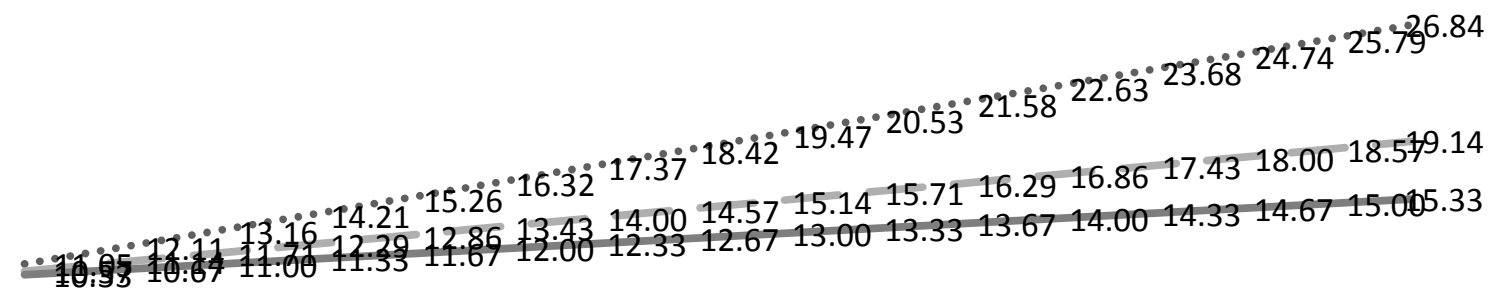

Lifting

$20 \quad 40$

60

80

$100 \quad 120$

140

16

180

$200 \quad 220 \quad 240 \quad 260$

280

(m)

Figure 2. Time needed to lift with respect to lifting height 


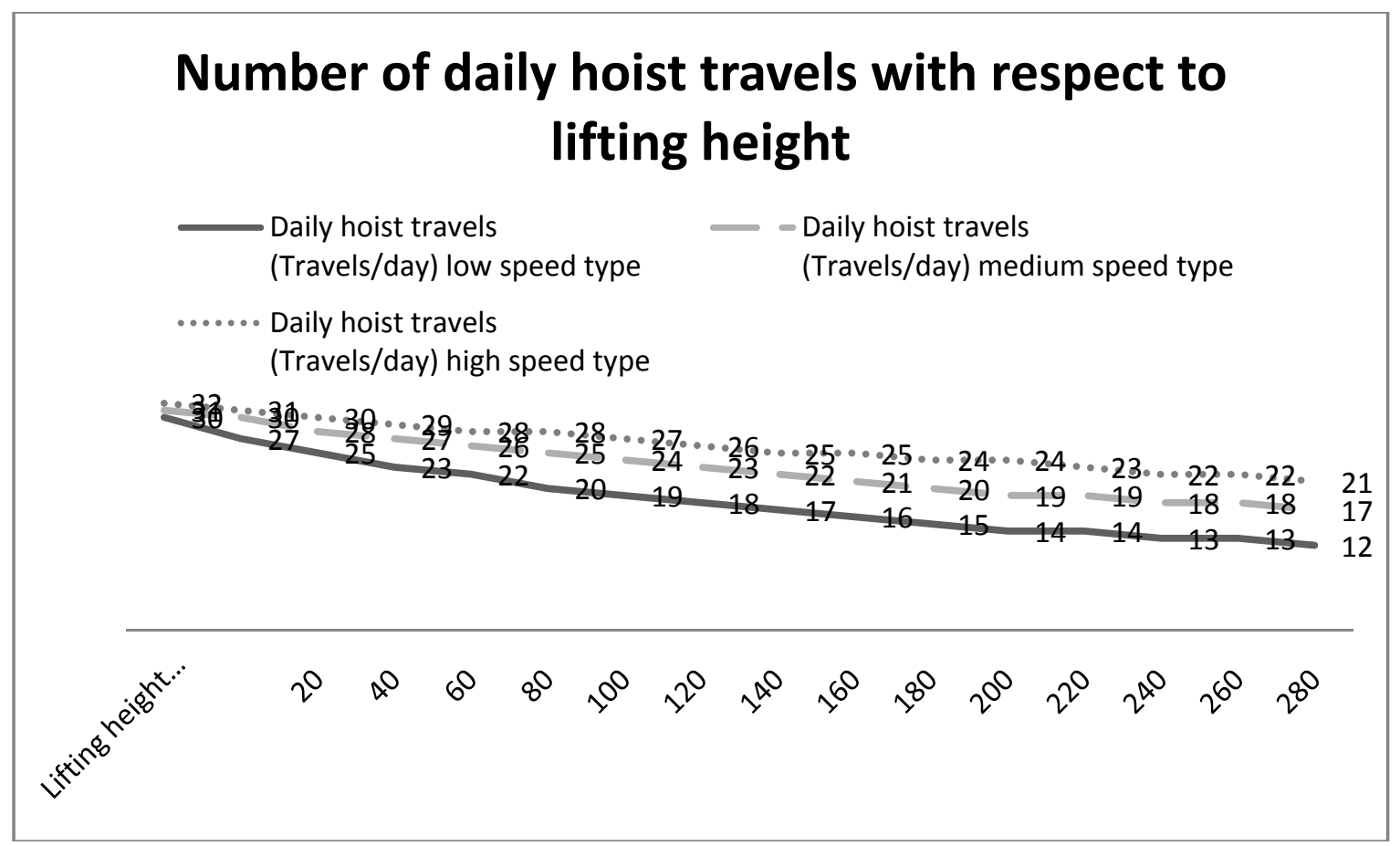

Figure 3. Number of daily hoist travels with respect to lifting height

According to Table 2, Figure 2 and Figure 3, number of hoist travels decreases as building height increases. For Burj Al Arab (321m), its daily travel would be limited to 22. For high-speed lifts

For different lead times ranging from 5 to 10 minutes, Table 3 and Figure 4 shows their effects.

Table 3. Sensitive Analysis For different lead times ranging from 5 to 10 minutes

\begin{tabular}{|c|c|c|c|c|c|c|}
\hline $\begin{array}{c}\text { Lifting } \\
\text { height }\end{array}$ & $\begin{array}{c}\text { leadtime } \\
10 \mathrm{~min}\end{array}$ & $\begin{array}{c}\text { leadtime } \\
9 \mathrm{~min}\end{array}$ & $\begin{array}{c}\text { Leadtime } \\
8 \mathrm{~min}\end{array}$ & $\begin{array}{c}\text { leadtime } \\
7 \mathrm{~min}\end{array}$ & $\begin{array}{c}\text { leadtime } \\
6 \mathrm{~min}\end{array}$ & $\begin{array}{c}\text { leadtime } \\
5 \mathrm{~min}\end{array}$ \\
\hline 20 & 32.52 & 36.00 & 40.32 & 45.82 & 53.05 & 63.00 \\
\hline 40 & 31.50 & 34.76 & 38.77 & 43.83 & 50.40 & 59.29 \\
\hline 60 & 30.55 & 33.60 & 37.33 & 42.00 & 48.00 & 56.00 \\
\hline 80 & 29.65 & 32.52 & 36.00 & 40.32 & 45.82 & 53.05 \\
\hline 100 & 28.80 & 31.50 & 34.76 & 38.77 & 43.83 & 50.40 \\
\hline 120 & 28.00 & 30.55 & 33.60 & 37.33 & 42.00 & 48.00 \\
\hline 140 & 27.24 & 29.65 & 32.52 & 36.00 & 40.32 & 45.82 \\
\hline 160 & 26.53 & 28.80 & 31.50 & 34.76 & 38.77 & 43.83 \\
\hline 180 & 25.85 & 28.00 & 30.55 & 33.60 & 37.33 & 42.00 \\
\hline 200 & 25.20 & 27.24 & 29.65 & 32.52 & 36.00 & 40.32 \\
\hline 220 & 24.59 & 26.53 & 28.80 & 31.50 & 34.76 & 38.77 \\
\hline 240 & 24.00 & 25.85 & 28.00 & 30.55 & 33.60 & 37.33 \\
\hline 260 & 23.44 & 25.20 & 27.24 & 29.65 & 32.52 & 36.00 \\
\hline 280 & 22.91 & 24.59 & 26.53 & 28.80 & 31.50 & 34.76 \\
\hline 300 & 22.40 & 24.00 & 25.85 & 28.00 & 30.55 & 33.60 \\
\hline 320 & 21.91 & 23.44 & 25.20 & 27.24 & 29.65 & 32.52 \\
\hline
\end{tabular}




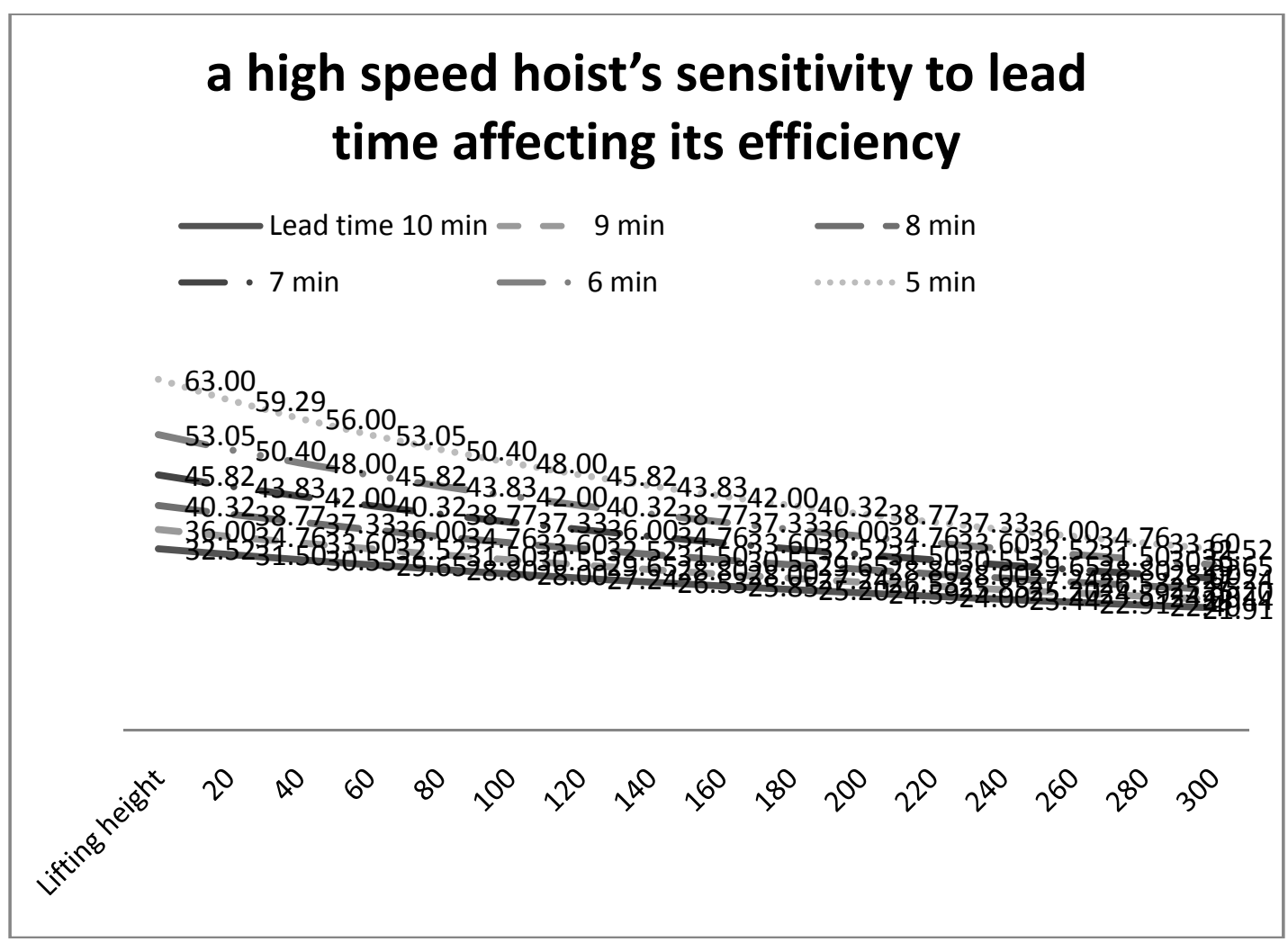

Figure 4. a high speed hoist's sensitivity to lead time affecting its efficiency

To improve the efficiency issues, substantial changes in existing hoist mechanism and their shape are obviously desirable; however, for seeking ready-to-use options, shrinking the lead time to the minimum seems to be a reasonable approach.

\subsection{Analysis of existing work process}

To understand the lead time in detail, we attempted to analyze the existing work process. We divide the workers related to hoist operation into two groups; the one for ground-level crews loading the hoist with materials, and the other for destination-level crews unloading the delivered materials. Figure 5 is a flow diagram of the work process.

In this figure, workflows of two work crews (ground-level crews and destination level ones) are clearly identified. At the ground-level floor, the crews would use the entrance door where as the destination crews would use the exit door.

The ten-minute lead time assumed in Table 2 corresponds to the 'stand-by' time in Figure 6, which implies that reduced crew-work would positively affect the stand-by time. Further breakdown of the workflow for both crew groups (and their time consumption) can be drawn as Figure 6 .

Let Tc1 (stand for Check Time) for the time needed for ground crew to identify total amount of the material to be loaded, Tl for the time needed to load the hoist. The stand-by time is then described as Tc1 + Tl. Likewise, Let Tc2 and Tu for the check time and time unloading time of the destination floor crews respectively. The stand-by time for the destination floor crew is described as Tc2 + Tu also.

Therefore, Total standby time (denoted as Ts) in a single lift cycle can be summarized in the following expression. Our intelligent hoist concept intends to reduce Ts, which will be further described in following chapters.

\section{Automation Technology Analysis}

\subsection{Available technologies}

Barcode systems have been used for supply chain management, which is being replaced by newer RFID 
systems. Bar code readers have shorter recognition ranges, with inability to scan multiple tags at a time, whereas RFIDs have longer ranges and simultaneous scanning capability.

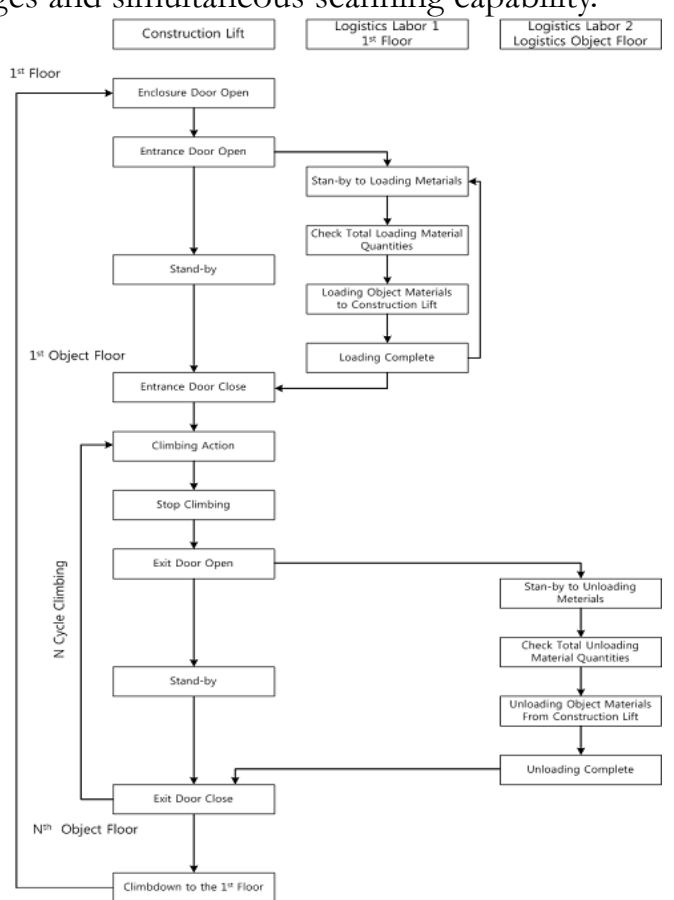

Figure 5. Flow diagram of As-is work process

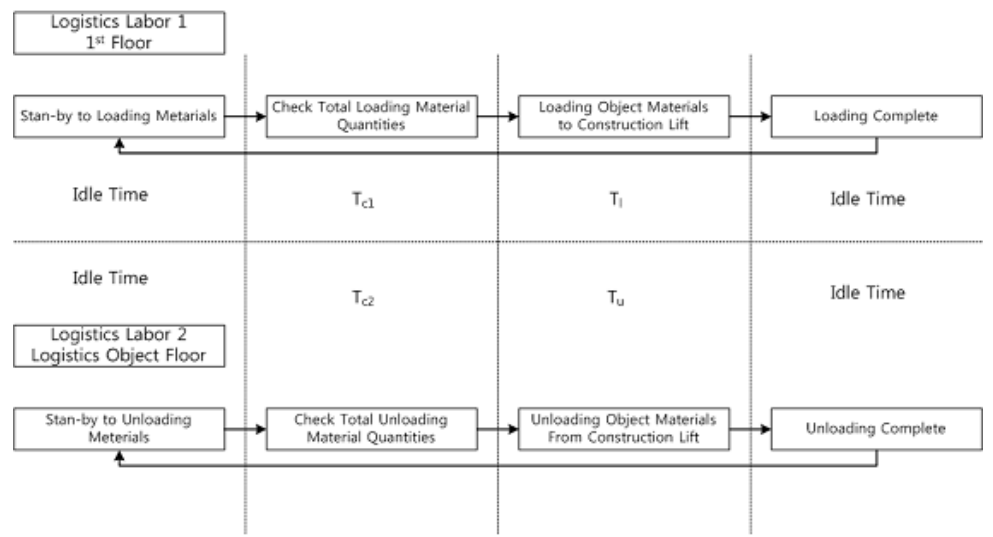

Figure 6. the workflow for both crew groups

In South Korean research society, RFIDs are explored with USN (Ubiquitous Sensor Network) for managing logistics in construction sites.

Among the listed researches, in the 'Next generation intelligent construction logistics automation system', now in its 3rd year, a toolkit for RFID material recognition, which is applicable to hoists as well enabling automatic request of the materials from each floor to the sensor-equipped hoist, is under development. The research also proved that the RFID-based system can interact with real-time construction project management system. The following Figure 7(Research report: An intelligent construction logistics automation system. Ministry of land, transportation, and maritime affairs) is a summary of this research. 


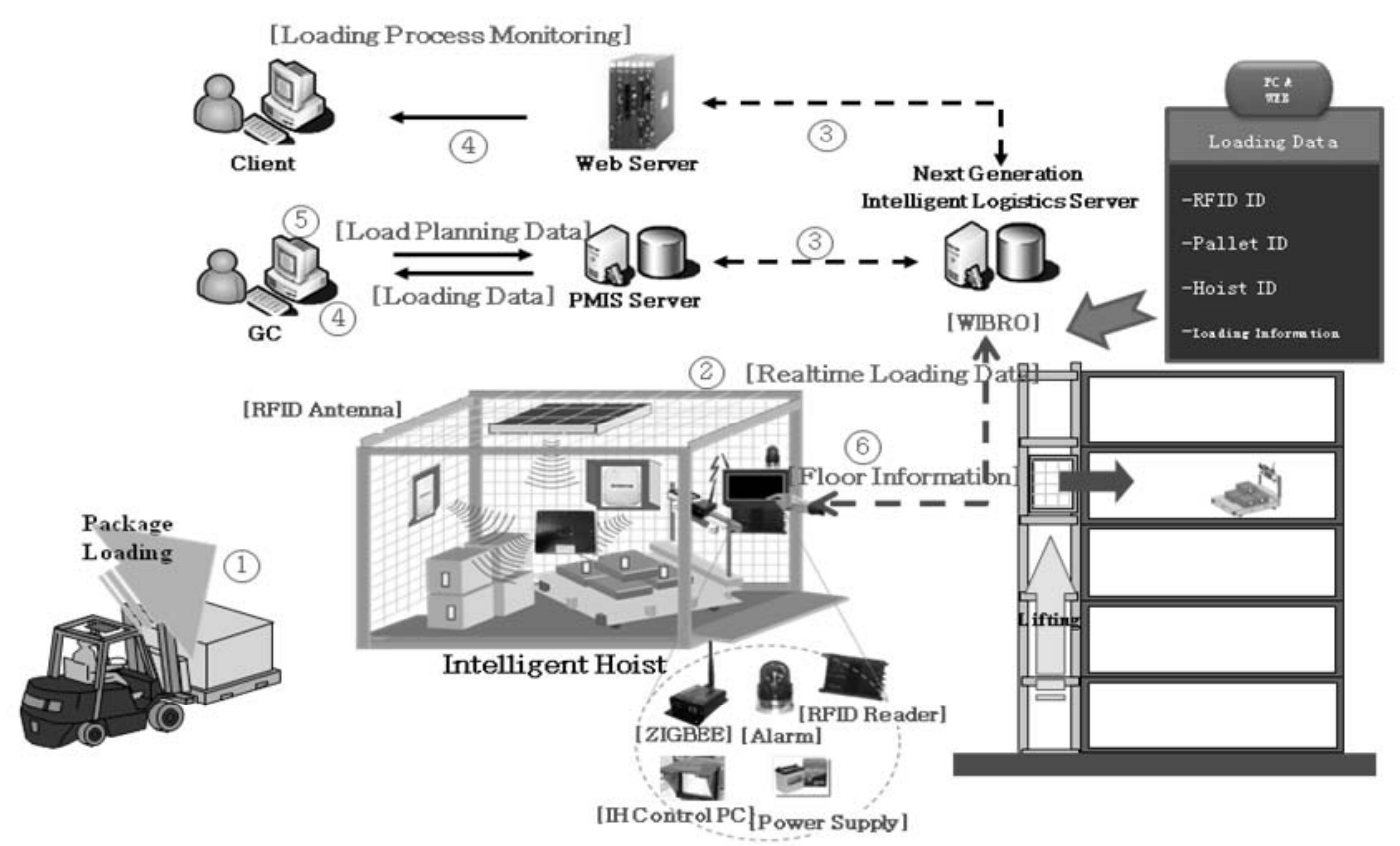

Figure 7. Development of Intelligent Construction Logistics System

Judging from these research cases, RFID is deemed to be feasible for replacing human labor regarding to checking materials prior to loading and unloading (i.e., Tc), thus reducing the check time.

First of all, the process of selecting the optimum tower cranes for super tall building projects such as collection of actual data, application of the data, decision-making support, etc. was delineated. Then, the function and logic for the development of the system for the selection of optimum tower cranes were analyzed, and the method for the application of the results of this study to the development of such a system was investigated.

\subsection{Smart Construction Lift Requirement for High Rise Building Construction}

Resulting from the research surveys and the work process study, we have defined the required functionalities of the smart construction lift for high-rise buildings, they are:

First, automated recognition of the destination floor for each material loaded to the hoist (with help of PMIS information)

Second, identifying material demands from each unloading floors

Third, performance for reducing loading/unloading time

We also laid out the possible approaches for implementing these requirements: The first requirement can be fulfilled with the development of a PMIS communication module. The second one is realized by RFID systems for sensing what the hoist currently has, besides by the PMIS communication for what needs to be unloaded to each floor. The third (and the last) one is possible by devising a new self-loadable end effector. The work process enhancements benefited from this projected development is illustrated in Figure 8.

\section{Conceptual Design}

\subsection{Conceptual design of Smart Construction Lift}

The smart construction lift is composed of the following modules, targeted for shorter stand-bys.

First, a self-loading module

Second, an RFID tag scanning module capable of identifying the loaded materials and their quantities.

Third, a communication module to get destination information (e.g. destination floor of given materials and their quantities) from PMIS.

Fourth, active floor recognition module for ever-varying floor information.

Fifth, a calculation module for travel optimization to a target floor

These modules and their functions are listed in the following figure. 


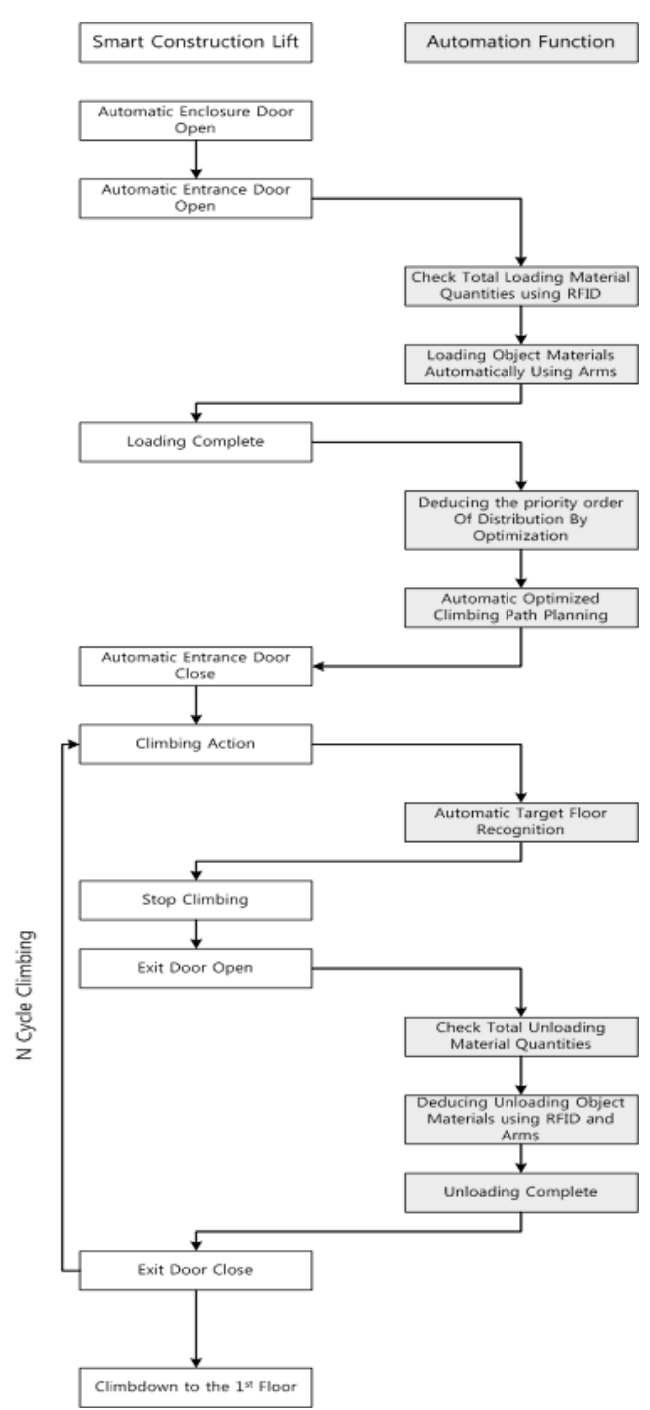

Figure 8. Work process enhancements benefited from this projected development

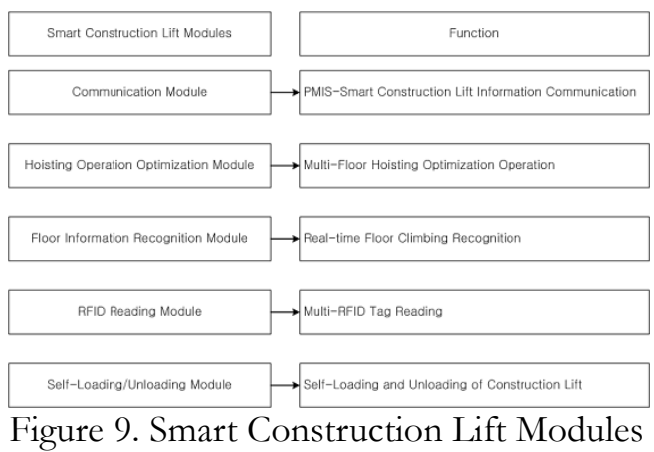

The data flow between the modules and the tasks performed by each module is illustrated in Figure 11.

\subsection{Proposed conceptual design for Smart Construction Lift}

For each module in Smart Construction Lift, they can be realized using the following technologies.

1. Communication modules can be realized using wireless communication technologies such as Mobile WiMax, WiFi, or Cellular network.

2. RFID modules can be implemented using the RFID readers and antennae

3. Floor recognition module can be implemented with an encoder, a pinion gear counter, and 
magnetic sensors.

4. Hoisting operation optimization module requires a control computer to calculate the optimized travel plan, which can be served with a personal computer

5. Self loading/unloading module can be implemented with an active hydraulic arm or a fork The configuration of the proposed modules is illustrated in Figure 12, forming a conceptual design of the Smart Construction Lift.

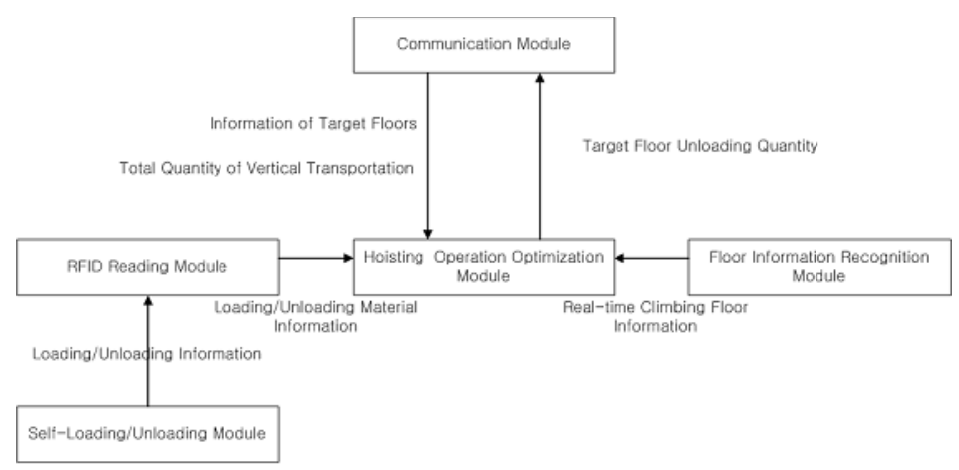

Figure 11. data flow between the modules and the tasks performed by each module

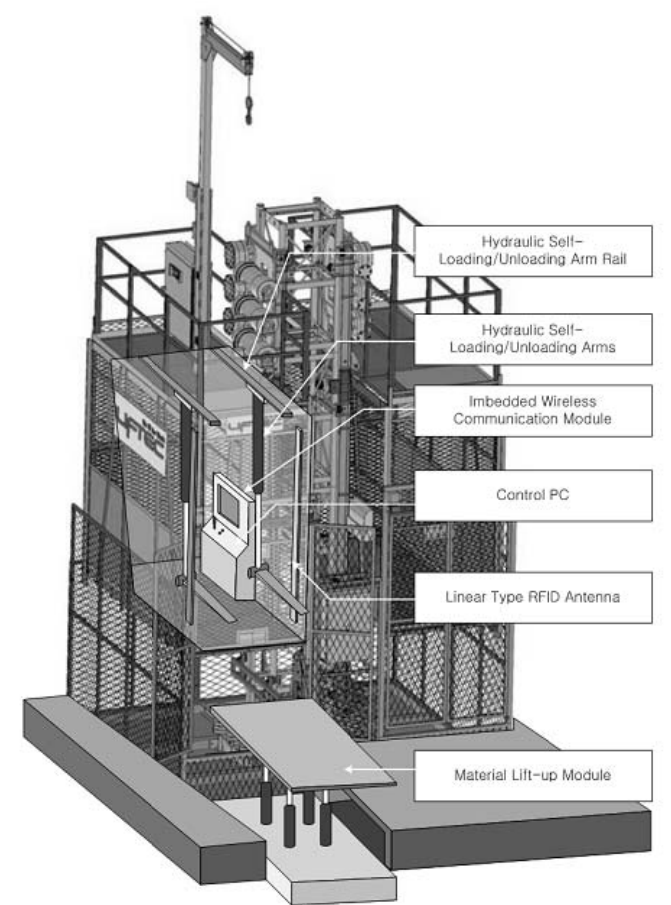

Figure 12. Conceptual Design of Smart Robotic Construction Lift

For implementing the hydraulic loading/unloading module, an fork type would be better if materials are conveyed on pallets and/or their quantities are big; for individual materials without pallets, arm types would be better.

For wireless communication modules, an embedded module (installed inside the control PC) is expected to fare well in terms of noise resistance rather than external one (hooked up via cable).

For RFID antennae, circular types seem better than linear types because construction lifts reflect radio waves causing interference

\subsection{Expected Benefits}

As mentioned in previous sections, this research aims to propose an alternative to conventional lifts for increased productivity without the need for completely redesigning them. For building projects where 
vertical transport accounts for significant amount of construction schedule, such benefit would be maximized.

The expected benefits of the proposed smart lift system can be summarized as Table 5, which is shown below.

Table 4. as-is logistic management matrix Table 5. To-be logistic management matrix

\begin{tabular}{|l|l|l|l|l|}
\hline & Labor ! & Labor 2 & Lft Operator & Lift \\
\hline loading packages at Storage Area & & & & \\
\hline moving packages to lift entrance & & & & \\
\hline Waiting for loading & & & & \\
\hline loading to lift & & & & \\
\hline Input target floor & & & & \\
\hline Lifting packages & & & & \\
\hline Fit lift-exit hight to flcor hight & & & & \\
\hline Open lift-exit & & & & \\
\hline unloadling & & & & \\
\hline close lift-exit & & & & \\
\hline lifting next target floo & & & & \\
\hline Fit lift-exit hight to flcor hight & & & & \\
\hline Open lift-exit & & & & \\
\hline unloading & & & & \\
\hline close lift-exit & & & \\
\hline
\end{tabular}

\begin{tabular}{|c|c|c|c|c|}
\hline & Labor 1 & Labor 2 & Lift Operator & Uift \\
\hline \multirow{2}{*}{\multicolumn{5}{|c|}{ loading padkages at Storage Area }} \\
\hline \multirow{2}{*}{\multicolumn{5}{|c|}{\begin{tabular}{|l} 
moving padxages to lift entrance \\
Waiting for loading
\end{tabular}}} \\
\hline & & & & \\
\hline loading to lift & 1 & & & \\
\hline \multicolumn{5}{|l|}{ Input target floor } \\
\hline \multicolumn{5}{|l|}{ Lufting padkages } \\
\hline Fit lift-exit hight to floor hight & & & & 3 \\
\hline \multicolumn{5}{|l|}{ Open liff-exit } \\
\hline \multicolumn{5}{|l|}{ unloading } \\
\hline \multicolumn{5}{|l|}{ close lift-ext } \\
\hline \multicolumn{5}{|l|}{ liffing next target floor } \\
\hline \multicolumn{5}{|l|}{ Fit lift-exit hight to floor hight } \\
\hline \multicolumn{5}{|l|}{ Open lift-exit } \\
\hline \multicolumn{5}{|l|}{ unloading } \\
\hline close Ifft-exit & & & & \\
\hline
\end{tabular}

- $1:$ automated loading system using hydraulic arms or folks

- 2 : automated system using RFID

- $\quad 3$ :automated floor sensing using light sensor and encoder

A to-be-developed hydraulic arms and forks will enable automatic loading and unloading (thus reducing labors needed for those operations). Also, overall lead time required prior to actual lifting can be reduced by various measures such as: utilizing RFID-based automatic logging, automatic identification of the destination floor of a given package with help of onboard control computer, identification of the lift's current location which will be implemented using light sensors and encoders. Such improvements will allow unmanned operation that won't require human operators, and they will enhance productivity of the storagearea operations thanks to shortened lead time.

\section{Conclusions and Further Studies}

The authors have proposed a concept of a smart construction lift, whose functionality can be summarized as follows:

First, automatic loading/unloading capability which enables unmanned operation.

Second, wireless communication with PMIS

Third, optimization capability in travel planning with help of PMIS information and automatic operation of the lift using the optimized plan

Fourth, automatic operation to the target floor.

Fifth, automatic unloading upon arrival at the target floor

Sixth, sending information about unloaded materials to PMIS in real time.

Once realized, the smart construction lift will help logistics in high-rise construction sites, where sending materials to higher locations often cause overall construction schedule delayed due to mechanical limits of the lifts. Further research plans include the prototype development and evaluation of it. Also, this research will contribute to the construction industry by demonstrating a cost-saving alternative to the revolutionary approaches to completely replace the existing rack-and-pinion mechanisms, which require substantially more money and time, besides the cost of 'trial-and-errors'.

\section{References}

[1] Research report: An intelligent construction logistics automation system. Ministry of land, transportation, and maritime affairs (Republic of Korea).

[2] Lee, J.B. et. al.(2008) A Feasibility Study on Optimal Lifting Planning in the High-rise Apartment Building Construction, Journal of Korea Institute of Construction Engineering and Management Institute 9(3), pp. 185-193 (Korean)

[3] Ho, J.K. (2007). A System for the Selection fo the Optimum Tower Cranes (Opt-TC). Journals of KICEM. 8(6). (Korean) 
[4] Kim, A.Y. (2007). A Basic Study on Selection of Climbing-type Crane and for Structuring the Stability Analysis Model. Proceeding of 13th Korean Institute of Ecological Architecture annual conference. (Korean)

[5] Kim, J.J. (2005). A Study on the Hoisting Planning System in Highrise Building Construction. Journal of Korea Institute of Construction 5(4). (Korean)

[6] Chung, Y.W. (2004). A study of the Highrise Building's Lift-up Management: Through Division of Loading Factors of Equipment Materials. Proceedings of 5th Annual Conference of KICEM. (Korean)

[7] Lee, H.S. (2004). A Study on the Method of New Activity Based Cost Management Coping With Changes in the Cost Structure of Real Estate Construction Industry. Journal of KICEM 5(1). (Korean)

[8] Kim, S.K. (2008). A Study on the Estimation of Proper Numbers of Construction Lifts. Journal of KIC 8(3). (Korean)

[9] Liftec Construction Lift Installation Manual. Pp.25. Liftec Inc, 2008.8 